\title{
Dual Curves of Constant Breadth in Dual Euclidean Space $\mathbb{D}^{3}$
}

\author{
Huseyin Kocayigit and and Nihal Erten \\ Department of Mathematics, Faculty of Science and Arts, Celal Bayar University, Manisa, Turkey
}

Received: 12 January 2016, Accepted: 25 January 2016

Published online: 19 June 2016.

\begin{abstract}
In this paper, we study dual curves of constant breadth in dual Euclidean Space $\mathbb{D}^{3}$. We obtain the differential equations characterizing dual curves of constant breadth in $\mathbb{D}^{3}$ and we introduce some special cases for these dual curves.
\end{abstract}

Keywords: Dual Euclidean space, dual Frenet frame, dual curve of constant breadth.

\section{Introduction}

The properties of plane convex curves have been studied by many geometers so far. Two brief reviews of the most important publications on this subject have been published by Struik [18]. Also, a number of interesting properties of plane curves of constant breadth are included in the works of Euler [8], Ball [2], Barbier [3], Blaschke [4] and Mellish [15].

A space curve of constant breadth was defined by Fujivara [9]. He considered a closed curve whose normal plane at a point $P$ has only one more point $Q$ in common with the curve, and for which $d(P, Q)$ is constant. For such curves $P Q$ is also normal at $Q$. Furthermore, spherical curve of constant breadth was defined by Blaschke [5]. Köse presented some concept for space curves of constant breadth in Euclidean 3-space [12,13]. Furthermore, differential equations characterizing space curves of constant breadth were obtained by Sezer [17]. Similar characterization of space curves of constant breadth in Euclidean 4-space were given by Mağden and Köse [14]. Also, the curves of constant breadth have been studied in Minkowski 3-space spacelike and timelike curves of constant breadth are normal curves, helices and spherical curves in some special cese. In [10], Kocayiğit and Önder showed that in Minkowski 3-space spacelike and timelike curves of constant breadth in are normal curves, helices and spherical curves in some special ceses. Moreover, Önder, Kocayiğit and Candan studied differential equations characterizing timelike and spacelike curves of constant breadth in Minkowski 3-space $E_{1}^{3}$ and gave a criterion for a timelike or spacelike curve of to be the curve of constant breadth in $E_{1}^{3}$ [16]. Spacelike curves of constant breadth in Minkowski 4-space were given by Kazaz, Önder and Kocayiğit [11]. Furthermore, Yılmaz have studied dual timelike curves of constant breadth in dual Lorentzian space [20].

In this paper we study dual curves of constant breadth in dual Lorentzian space $\mathbb{D}^{3}$. We obtain a third order differential equation which characterizes dual curves of constant breadth in $\mathbb{D}^{3}$ and give some special cases. 


\section{Dual curves and dual Euclidean space}

Let $\mathbb{D}=\mathbb{R} \times \mathbb{R}=\left\{\bar{a}=\left(a, a^{*}\right): a, a^{*} \in \mathbb{R}\right\}$ be the set of the pairs $\left(a, a^{*}\right)$.For $\bar{a}=\left(a, a^{*}\right), \bar{b}=\left(b, b^{*}\right) \in \mathbb{D}$ the following operations are defined on $\mathbb{D}$ :

$$
\begin{aligned}
& \text { Equality: } \bar{a}=\bar{b} \Leftrightarrow a=b, a^{*}=b^{*} \\
& \text { Addition }: \bar{a}+\bar{b}=\left(a+b, a^{*}+b^{*}\right) \\
& \text { Multiplication }: \bar{a} \bar{b}=\left(a b, a b^{*}+a^{*} b\right) .
\end{aligned}
$$

The element $\varepsilon=(0,1) \in \mathbb{D}$ satisfies the relationships

$$
\varepsilon \neq 0, \quad \varepsilon^{2}=0, \quad \varepsilon 1=1 \varepsilon=\varepsilon
$$

Let consider the element $\bar{a} \in \mathbb{D}$ of the form $\bar{a}=(a, 0)$. Then the mapping $f: \mathbb{D} \rightarrow \mathbb{R}, f(a, 0)=a$ is a isomorphism. So, we can write $a=(a, 0)$. By the multiplication rule we have that

$$
\bar{a}=\left(a, a^{*}\right)=(a, 0)+\left(0, a^{*}\right)=(a, 0)+(0,1)\left(a^{*}, 0\right)=a+\varepsilon a^{*}
$$

Then $\bar{a}=a+\varepsilon a^{*}$ is called dual number and $\varepsilon$ is called dual unit. Thus the set of dual numbers is given by

$$
\mathbb{D}=\left\{\bar{a}=a+\varepsilon a^{*}: a, a^{*} \in \mathbb{R}, \varepsilon^{2}=0\right\}
$$

The set $\mathbb{D}$ forms a commutative group under addition [6,7]. The associative laws hold for multiplication. Dual numbers are distributive and form a ring over the real number field.

Dual function of dual number presents a mapping of a dual numbers space on itself. The general expression for dual analytic (differentiable) function as follows

$$
f(\bar{x})=f\left(x+\varepsilon x^{*}\right)=f(x)+\varepsilon x^{*} f^{\prime}(x),
$$

where $f^{\prime}(x)$ is derivative of $f(x)$ and $x, x^{*} \in \mathbb{R}$. Let $\mathbb{D}^{3}=\mathbb{D} \times \mathbb{D} \times \mathbb{D}$ be the set of all triples of dual numbers, i.e.,

$$
\mathbb{D}^{3}=\left\{\tilde{a}=\left(\bar{a}_{1}, \bar{a}_{2}, \bar{a}_{3}\right): \bar{a}_{i} \in \mathbb{D}, i=1,2,3\right\} .
$$

Then the set $\mathbb{D}^{3}$ is called dual space. The elements of $\mathbb{D}^{3}$ are called dual vectors. Similar to the dual numbers, a dual vector $\tilde{a}$ may be expressed in the form $\tilde{a}=\mathbf{a}+\varepsilon \mathbf{a}^{*}=\left(\mathbf{a}, \mathbf{a}^{*}\right)$, where $\mathbf{a}$ and $\mathbf{a}^{*}$ are the vectors of $\mathbb{R}^{3}$. Then for any vectors $\tilde{a}=\mathbf{a}+\varepsilon \mathbf{a}^{*}$ and $\tilde{b}=\mathbf{b}+\varepsilon \mathbf{b}^{*}$ of $\mathbb{D}^{3}$, the scalar product and the vector product are defined by

$$
<\tilde{a}, \tilde{b}>=<\mathbf{a}, \mathbf{b}>+\varepsilon\left(<\mathbf{a}, \mathbf{b}^{*}>+<\mathbf{a}^{*}, \mathbf{b}>\right),
$$

and

$$
\tilde{a} \times \tilde{b}=\mathbf{a} \times \mathbf{b}+\varepsilon\left(\mathbf{a} \times \mathbf{b}^{*}+\mathbf{a}^{*} \times \mathbf{b}\right),
$$

respectively, where $\langle\mathbf{a}, \mathbf{b}\rangle$ and $\mathbf{a} \times \mathbf{b}$ are the inner product and the vector product of the vectors $\mathbf{a}$ and $\mathbf{b}$ in $\mathbb{R}^{3}$, respectively.

The norm of a dual vector $\tilde{a}$ is given by

$$
\|\tilde{a}\|=\|\mathbf{a}\|+\varepsilon \frac{<\mathbf{a}, \mathbf{a}^{*}>}{\|\mathbf{a}\|},(\mathbf{a} \neq 0)
$$

A dual vector $\tilde{a}$ with norm $1+\varepsilon 0$ is called dual unit vector. The set of dual unit vectors is given by 


$$
\tilde{S}^{2}=\left\{\tilde{a}=\left(a_{1}, a_{2}, a_{3}\right) \in \mathbb{D}^{3}:<\tilde{a}, \tilde{a}>=1+\varepsilon 0\right\},
$$

and called dual unit sphere (For details [6,7,19]).

Let $\tilde{\phi}: \mathbf{I} \subset \mathbb{R} \rightarrow \mathbb{D}_{1}^{3}$ be a dual curve with are lenght parameter $\bar{s}$. Then the unit tangent vector is defined $\dot{\tilde{\phi}}=\frac{d \tilde{\phi}}{d \bar{s}}=\tilde{t}$ and the principal normal is $\tilde{n}=\frac{\dot{t}}{\bar{\kappa}}$ where $\bar{\kappa}$ is never pure-dual and "." denotes the derivative with respect to $\bar{s}$. The function $\bar{\kappa}(\bar{s})=\|\tilde{t}\|=\kappa(s)+\varepsilon \kappa^{*}(s)$ is called dual curvature of dual curve $\tilde{\phi}$. Then the binormal vector of $\tilde{\phi}$ is given by the dual vector $\tilde{b}=\tilde{t} \times \tilde{n}$. Hence, the triple $\{\tilde{t}, \tilde{n}, \tilde{b}\}$ is called dual Frenet frame of $\tilde{\phi}$ and Frenet formulas are given by

$$
\left[\begin{array}{c}
\dot{\tilde{t}} \\
\dot{\tilde{n}} \\
\dot{\tilde{b}}
\end{array}\right]=\left[\begin{array}{ccc}
0 & \bar{\kappa} & 0 \\
-\bar{\kappa} & 0 & \bar{\tau} \\
0 & -\bar{\tau} & 0
\end{array}\right]\left[\begin{array}{c}
\tilde{t} \\
\tilde{n} \\
\tilde{b}
\end{array}\right]
$$

where $\langle\tilde{t}, \tilde{t}>=1,<\tilde{n}, \tilde{n}>=1,<\tilde{b}, \tilde{b}>=1$ and $\langle\tilde{t}, \tilde{n}>=<\tilde{t}, \tilde{b}>=<\tilde{n}, \tilde{b}>=0$.

Here $\bar{\tau}(\bar{s})=\tau(s)+\varepsilon \tau^{*}(s)$ is dual torsion of dual curve $\tilde{\phi}$ and we suppose, as the curvature $\bar{\tau}$ is never pure-dual [1].

\section{Dual curves of constant breadth in $\mathbb{D}^{3}$}

In this section, we consider dual curves of constant breadth in dual Euclidean space $\mathbb{D}^{3}$.

Definition 1. Let $C$ be a dual curve in $\mathbb{D}^{3}$ with dual position vector $\tilde{\phi}=\tilde{\phi}(\bar{s})$. If $C$ has parallel tangents in opposite directions at corresponding points and if the distance between these points is always constant then $C$ is called a dual curve of constant breadth in $\mathbb{D}^{3}$. Moreover, a pair of dual curves $C$ and $C_{\zeta}$ for which the tangents at the corresponding points $\tilde{\phi}(\bar{s})$ and $\tilde{\zeta}\left(\bar{\zeta}_{\zeta}\right)$ are parallel and in opposite directions and the distance between these points is always constant is called a dual curve pair of constant breadth in $\mathbb{D}^{3}$ where $\tilde{\zeta}(\overline{\zeta \zeta})$ is dual position vector of dual curve $C_{\zeta}$ in $\mathbb{D}^{3}$.

Let now $C$ and $C_{\zeta}$ be a pair of unit speed dual curves of class $C^{3}$ with nonzero dual curvature and dual torsion in $\mathbb{D}^{3}$ and let those dual curves have parallel tangents in opposite directions at corresponding points. Then we may write the position vector of dual curve $C_{\zeta}$ as follows

$$
\tilde{\zeta}=\tilde{\phi}+\bar{\gamma} \tilde{t}+\bar{\delta} \tilde{n}+\bar{\lambda} \tilde{b}
$$

where $\bar{\gamma}, \bar{\delta}$ and $\bar{\lambda}$ are arbitrary functions of $\bar{s}$. By differentiating (2) with respect to $\bar{s}$ we get

$$
\frac{d \tilde{\zeta}}{d \bar{s}_{\zeta}} \frac{d \bar{s}_{\zeta}}{d \bar{s}}=\left(1+\frac{d \bar{\gamma}}{d \bar{s}}-\bar{\kappa} \bar{\delta}\right) \tilde{t}+\left(\bar{\kappa} \bar{\gamma}+\frac{d \bar{\delta}}{d \bar{s}}-\bar{\tau} \bar{\lambda}\right) \tilde{n}+\left(\bar{\tau} \bar{\delta}+\frac{d \bar{\lambda}}{d \bar{s}}\right) \tilde{b}
$$

Since we have $\tilde{t}_{\zeta}=-\tilde{t}$ from (3) it follows

$$
\left\{\begin{array}{l}
\frac{d \bar{\gamma}}{d \bar{s}}=-1-\frac{d \bar{s}_{\zeta}}{d \bar{s}}+\bar{\kappa} \bar{\delta} \\
\frac{d \bar{\delta}}{d \bar{s}}=-\bar{\kappa} \bar{\gamma}+\bar{\tau} \bar{\lambda} \\
\frac{d \bar{\lambda}}{d \bar{s}}=-\bar{\tau} \bar{\delta}
\end{array}\right.
$$

If we call $\bar{\varphi}$ as the dual angle between the tangent of dual curve $C$ at the point $\tilde{\phi}$ and a given fixed direction and consider $\frac{d \bar{\varphi}}{d \bar{s}}=\bar{\kappa}=\frac{1}{\bar{\rho}}$ and $\frac{d \bar{\varphi}}{d \bar{s}_{\zeta}}=\bar{\kappa}_{\zeta}=\frac{1}{\bar{\rho}_{\zeta}}$ then the system (4) can be given as follows 


$$
\left\{\begin{array}{l}
\frac{d \bar{\gamma}}{d \bar{\varphi}}=-\bar{\delta}-f(\bar{\varphi}) \\
\frac{d \bar{\delta}}{d \bar{\varphi}}=-\bar{\gamma}+\bar{\tau} \bar{\rho} \bar{\lambda} \\
\frac{d \bar{\lambda}}{d \bar{\varphi}}=-\bar{\tau} \bar{\rho} \bar{\delta}
\end{array}\right.
$$

where $f(\bar{\varphi})=\bar{\rho}+\bar{\rho}_{\zeta}$. Using the system of ordinary differential equations (5), we have the following dual third order differential equation with respect to $\bar{\gamma}$

$$
\left\{\frac{d}{d \bar{\varphi}}\left[\frac{\bar{\kappa}}{\bar{\tau}}\left(\frac{d f}{d \bar{\varphi}}+\frac{d^{2} f}{d \bar{\varphi}^{2}}\right)\right]\right\}+\frac{\bar{\tau}}{\bar{\kappa}}\left(f+\frac{d \bar{\gamma}}{d \bar{\varphi}}\right)-\frac{d}{d \bar{\varphi}}\left(\frac{\bar{\kappa}}{\bar{\tau}} \bar{\gamma}\right)=0
$$

Then we can give the following corollary:

Corollary 1. The dual differential equation given in (6) is a characterization for dual curve $C_{\zeta}$ and the position vector of this dual curve can be determined by the solution of this equation.

Let now investigate the solution of equation (6) in a special case. Assume that $\bar{\kappa}(\bar{s}), \bar{\tau}(\bar{s})$ be constants i.e., $\kappa, \kappa^{*}, \tau$ and $\tau^{*}$ be constant. It means that dual curve $C$ is a dual helix. In the case equation (6) has the form

$$
\frac{d^{3} \bar{\gamma}}{d \bar{\varphi}^{3}}+\left[1+\left(\frac{\bar{\tau}}{\bar{\kappa}}\right)^{2}\right] \frac{d \bar{\gamma}}{d \bar{\varphi}}+f(\bar{\varphi})=0
$$

Then we have the following corollary:

Corollary 2. The dual differential equation characterizing dual helix curves of constant breadth in $\mathbb{D}^{3}$ is given as follows

$$
\frac{d^{3} \bar{\gamma}}{d \bar{\varphi}^{3}}+\left[1+\left(\frac{\bar{\tau}}{\bar{\kappa}}\right)^{2}\right] \frac{d \bar{\gamma}}{d \bar{\varphi}}+f(\bar{\varphi})=0
$$

Corollary 3. Let C be a dual helix. In this case equation (7) gives us followings

$$
\left\{\begin{array}{l}
\bar{\gamma}=A \cos \left(\bar{\varphi} \sqrt{\frac{\bar{\tau}^{2}}{\bar{\kappa}^{2}}+1}\right)+B \sin \left(\bar{\varphi} \sqrt{\frac{\bar{\tau}^{2}}{\bar{\kappa}^{2}}+1}\right)-\frac{\bar{\kappa}^{2} \bar{\varphi}}{\bar{\kappa}^{2}+\bar{\tau}^{2}} f \\
\bar{\delta}=\sqrt{\frac{\bar{\tau}^{2}}{\bar{\kappa}^{2}}+1}\left[B \cos \left(\bar{\varphi} \sqrt{\frac{\bar{\tau}^{2}}{\bar{\kappa}^{2}}+1}\right)-A \sin \left(\bar{\varphi} \sqrt{\frac{\bar{\tau}^{2}}{\bar{\kappa}^{2}}+1}\right)\right]+\frac{\bar{\tau}^{2}}{\bar{\kappa}^{2}+\bar{\tau}^{2}} f \\
\bar{\lambda}=-\frac{\bar{\tau}}{\bar{\kappa}}\left[A \cos \left(\bar{\varphi} \sqrt{\frac{\bar{\tau}^{2}}{\bar{\kappa}^{2}}+1}\right)+B \sin \left(\bar{\varphi} \sqrt{\frac{\bar{\tau}^{2}}{\bar{\kappa}^{2}}+1}\right)\right]-\frac{1}{\bar{\tau}}\left(\frac{\bar{\kappa}^{2} \bar{\varphi}}{\bar{\kappa}^{2}+\bar{\tau}^{2}} f\right)
\end{array}\right.
$$

where $A$ and $B$ are constants. Then we have the following theorem.

Theorem 1. Dual helices $C$ and $C_{\zeta}$ form a dual curve pair of constant breadth in $\mathbb{D}^{3}$ if and only if there exists the following relationship between the dual position vectors of the curves 


$$
\begin{aligned}
& \tilde{\zeta}=\tilde{\phi}+\left[A \cos \left(\bar{\varphi} \sqrt{\frac{\bar{\tau}^{2}}{\bar{\kappa}^{2}}+1}\right)+B \sin \left(\bar{\varphi} \sqrt{\frac{\bar{\tau}^{2}}{\bar{\kappa}^{2}}+1}\right)-\frac{\bar{\kappa}^{2} \bar{\varphi}}{\bar{\kappa}^{2}+\bar{\tau}^{2}} f\right] \tilde{t} \\
& +\left[\sqrt{\frac{\bar{\tau}^{2}}{\bar{\kappa}^{2}}+1}\left[B \cos \left(\bar{\varphi} \sqrt{\frac{\bar{\tau}^{2}}{\bar{\kappa}^{2}}+1}\right)-A \sin \left(\bar{\varphi} \sqrt{\frac{\bar{\tau}^{2}}{\bar{\kappa}^{2}}+1}\right)\right]+\frac{\bar{\tau}^{2}}{\bar{\kappa}^{2}+\bar{\tau}^{2}} f\right] \tilde{n} \\
& +\left[-\frac{\bar{\tau}}{\bar{\kappa}}\left[A \cos \left(\bar{\varphi} \sqrt{\frac{\bar{\tau}^{2}}{\bar{\kappa}^{2}}+1}\right)+B \sin \left(\bar{\varphi} \sqrt{\frac{\bar{\tau}^{2}}{\bar{\kappa}^{2}}+1}\right)\right]-\frac{1}{\bar{\tau}}\left(\frac{\bar{\kappa}^{3} \bar{\varphi}}{\bar{\kappa}^{2}+\bar{\tau}^{2}} f\right)\right] \tilde{b} .
\end{aligned}
$$

Let now consider the general case again, i.e., let $\kappa, \kappa^{*}, \tau$ and $\tau^{*}$ be non-constant. Since the distance between opposite points of $C$ and $C_{\zeta}$ is constant, we can write

$$
\|\tilde{\zeta}-\tilde{\phi}\|^{2}=|\tilde{\gamma}+\tilde{\delta}+\tilde{\lambda}|=\text { constant } .
$$

By differentiating (9) with respect to $\bar{\varphi}$ it follows

$$
\bar{\gamma} \frac{d \bar{\gamma}}{d \bar{\varphi}}+\bar{\delta} \frac{d \bar{\delta}}{d \bar{\varphi}}+\bar{\lambda} \frac{d \bar{\lambda}}{d \bar{\varphi}}=0
$$

By virtue of (5), the differential equation (10) yields

$$
\bar{\gamma}\left(\frac{d \bar{\gamma}}{d \bar{\varphi}}-\bar{\delta}\right)=0 .
$$

From (11) the following cases are obtained.

(i) $\bar{\gamma}=0$. Then, from (5) the other components are

$$
\bar{\delta}=f, \quad \bar{\lambda}=\frac{\bar{\kappa}}{\bar{\tau}} \frac{d f}{d \bar{\varphi}}
$$

and we get

$$
\tilde{\zeta}=\tilde{\phi}+f \tilde{n}+\frac{\bar{\kappa}}{\bar{\tau}} \frac{d f}{d \bar{\varphi}} \tilde{b}
$$

(ii) $\frac{d \bar{\gamma}}{d \bar{\varphi}}=\bar{\delta}$. That is $f=0$. Then followings are obtained

(a) $\kappa=\tau$ and $\kappa^{*}=\tau^{*}$. Thus, the components are

$$
\left\{\begin{array}{l}
\bar{\gamma}=A \cos (\sqrt{2} \bar{\varphi})+B \sin (\sqrt{2} \bar{\varphi}) \\
\bar{\delta}=\sqrt{2}(-A \sin (\sqrt{2} \bar{\varphi})+B \cos (\sqrt{2} \bar{\varphi})) \\
\bar{\lambda}=-(A \cos (\sqrt{2} \bar{\varphi})+B \sin (\sqrt{2} \bar{\varphi}))
\end{array}\right.
$$

(b) $\frac{\kappa}{\tau}=\frac{\kappa^{*}}{\tau^{*}}, \tau>\kappa$ and $1+\frac{\bar{\tau}^{2}}{\bar{\kappa}^{2}}$. Then the components

$$
\left\{\begin{array}{l}
\bar{\gamma}=A \cos \left(\sqrt{1+\frac{\bar{\tau}^{2}}{\bar{\kappa}^{2}}} \bar{\varphi}\right)+B \sin \left(\sqrt{1+\frac{\bar{\tau}^{2}}{\bar{\kappa}^{2}}} \bar{\varphi}\right) \\
\bar{\delta}=\sqrt{1+\frac{\bar{\tau}^{2}}{\overline{\bar{\kappa}}^{2}}}\left[-A \cos \left(\sqrt{1+\frac{\bar{\tau}^{2}}{\overline{\bar{\kappa}}^{2}}} \bar{\varphi}\right) B \sin \left(\sqrt{1+\frac{\bar{\tau}^{2}}{\overline{\bar{\kappa}}^{2}}} \bar{\varphi}\right)\right] \\
\bar{\lambda}=\frac{\tau}{\kappa}\left[A \cos \left(\sqrt{1+\frac{\bar{\tau}^{2}}{\bar{\kappa}^{2}}} \bar{\varphi}\right)+B \sin \left(\sqrt{1+\frac{\bar{\tau}^{2}}{\bar{\kappa}^{2}}} \bar{\varphi}\right)\right]
\end{array}\right.
$$




\section{Conclusion}

In this study, some characterizations of space curves according to Bishop frame in Euclidean 3-space are given by using Laplacian operator and Levi-Civita connection. Furthermore, the general differential equations which characterize the space curves according to Bishop frame are given. It is obtained some useful results and given theorems. Similar characterizations can be applied to other curves.

\section{References}

[1] Ayyıldız, N., Çöken, A.C., Yücesan, A., A Characterization of Dual Lorentzian Spherical Curves in the Dual Lorentzian Space, Taiwanese Journal of Mathematics, 11(4), 999-1018 (2007).

[2] Ball, N. H., On Ovals, American Mathematical Monthly, 37(7), 348-353 (1930).

[3] Barbier, E., Note sur le probleme de l'aiguille et le jeu du joint couvert. J. Math. Pures Appl., II. Ser. 5, $273-286$ (1860).

[4] Blaschke, W., Konvexe bereiche gegebener konstanter breite und kleinsten inhalts, Mathematische Annalen, B. 76(4), 504-513 (1915).

[5] Blaschke, W., Einige Bemerkungen über Kurven und Flächen konstanter Breite. Ber. Verh. Sächs. Akad. Leipzig, 67, 290-297 (1915).

[6] Blaschke, W., Differential Geometric and Geometrischke Grundlagen ven Einsteins Relativitasttheorie Dover, New York, (1945).

[7] Hacısalihoğlu, H.H., Hareket Geometrisi ve Kuaterniyonlar Teorisi, Gazi Üniversitesi Fen-Edb. Fakültesi, (1983)

[8] Euler, L., De Curvis Triangularibus, Acta Acad. Petropol., 3-30 (1778-1780).

[9] Fujivara, M., On space curves of constant breadth, Tohoku Math., J., 5, 180-184 (1914).

[10] Kocayiğit, H., Önder, M., Space curves of constant breadth in Minkowski 3-space, Annali di Matematica, 192(5), 805-814, (2013).

[11] Kazaz, M., Önder, M., Kocayiğit, H., Spacelike curves of constant breadth in Minkowski 4-space, Int. Journal of Math. Analysis, 22(2) 1061-1068 (2008).

[12] Köse, Ö., On space curves of constant breadth, Doğa Tr. J. Math., 10(1), 11-14, (1986).

[13] Köse, Ö., Some Properties of Ovals and Curves of Constant Width in a Plane, Doğa Mat., 2 (8) 119-126 (1984).

[14] Mağden, A., Köse, Ö., On the curves of constant breadth in $E^{4}$ space, Turkish J. Math., 21 (3) 277-284 (1997).

[15] Mellish, A.P., Notes of Differential Geometry, Annals of Mathematics, 32(1), 181-190 (1931).

[16] Önder, M., Kocayiğit, H., Candan, E., Differential Equations Characterizing Timelike and Spacelike Curves of Constant Breadth in Minkowski 3-Space $E_{1}^{3}$, J. Korean Math. Soc. 48 (4) 849-866 (2011).

[17] Sezer, M., Differential equations characterizing space curves of constant breadth and a criterion for these curves, Turkish J. of Math. 13(2), 70-78 (1989).

[18] Struik, D. J., Differential Geometry in the Large, Bull. Amer. Math. Soc., 37(2), 49-62 (1931).

[19] Veldkamp, G.R., On the use of dual numbers, vectors and matrices in instantaneous spatial kinematics, Mech. Mach. Theory, 11(2), 141-156 (1976).

[20] Yilmaz, S., Time-like Dual Curves of Constant Breadth in Dual Lorentzian Space, IBSU Scientific Journal, 2 (2), 129-136 (2008). 